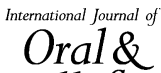 \\ Maxillofacial \\ Surgery \\ Clinical Paper \\ Head and Neck Oncology
}

\section{Do we have a robust method for preoperative tumour depth assessment for oral cavity tumours with clinically negative necks?}

H. Cocker, O. Francies, A. Adams, I. Sassoon, C. Schilling: Do we have a robust method for preoperative tumour depth assessment for oral cavity tumours with clinically negative necks?. Int. J. Oral Maxillofac. Surg. 2019; xxx: xxx-xxx. (C) 2020 International Association of Oral and Maxillofacial Surgeons. Published by Elsevier Ltd. All rights reserved.

Abstract. Tumour depth is an important prognostic factor in head and neck cancer and has recently been included in the eighth edition of the Union for International Cancer Control TNM classification of malignant tumours for oral squamous cell carcinoma (OSCC). It is important to appraise the accuracy of depth assessments; however, there is little current evidence in the literature. Accurate depth assessment is particularly pertinent in cT1-T2N0 OSCC where it may influence neck management. A retrospective study was performed at two tertiary referral centres, in which surgically treated patients with cT1-T4N0 OSCC were audited. Preoperative tumour depth assessments from multimodality radiological staging scans were compared with the final histopathological depth. The predictive accuracy of intraoral ultrasound (IOUS), computed tomography (CT), and magnetic resonance imaging (MRI) for tumour depth was evaluated. Accuracy to within $3 \mathrm{~mm}$ of the histopathological depth was seen in $56.7 \%$ of MRI scans and $57.1 \%$ of CT scans. IOUS appeared to have superior prediction, with $78.2 \%$ of measurements within $3 \mathrm{~mm}$. Over one third of CT and MRI imaging failed to detect a lesion; IOUS scans detected the lesions in all of these case. In conclusion, the reliability of preoperative imaging assessment of tumour depth should be considered when recommending treatment.
H. Cocker, O. Francies, A. Adams, I. Sassoon, C. Schilling

University College London Hospital, Bloomsbury, London, UK
Key words: neoplasms; mouth; squamous cell carcinoma of head and neck; neoplasm staging; neoplasm invasiveness. 
Tumour depth is a prognostic factor in head and neck cancer, positively correlated with both the risk of occult nodal disease and local recurrence ${ }^{1-3}$.

The International Consortium for Outcome Research (ICOR) in head and neck cancer investigated the predictive value of depth of invasion ${ }^{3}$, and this led to the inclusion of tumour depth as an independent variable in the new Union for International Cancer Control (UICC) staging criteria (eighth edition of the TNM classification of malignant tumours, TNM $8^{1}$ ).

Although the importance of tumour depth is widely recognized, there is no consensus yet over the use of critical tumour depth to recommend a change in treatment. This debate has included cutoff points ranging from $1.5 \mathrm{~mm}$ to $10 \mathrm{~mm}$ to decide whether patients should be offered elective neck surgery despite radiographic N0 staging ${ }^{1-7}$.

Huang et al. ${ }^{8}$ showed tumour depth of $>4 \mathrm{~mm}$ to be statistically significant in predicting occult neck metastases and advised elective treatment of the neck in such cases. Furthermore, recent National Comprehensive Cancer Network (NCCN) guidelines have included the same cut-off for consideration of elective neck dissection (END) in the cN0 neck ${ }^{9}$. However, treatment recommendations based on preoperative tumour depth can only be effective if we have a reliable method of assessing tumour dimensions preoperatively.

The purpose of this study was to determine whether current methods to assess tumour depth preoperatively are accurate when compared to postoperative histopathology specimens, for patients with oral squamous cell carcinoma (OSCC) and clinically N0 necks.

\section{Materials and methods}

This multicentre retrospective study was performed between 2014 and 2018 and included patients seen at head and neck multidisciplinary team (MDT) meetings, who had oral cavity tumours staged N0 by the nationally recommended staging imaging protocol ${ }^{2,10}$. Results from two centres (MDT1 and MDT2) were combined for analysis; these were approved and registered by the clinical effectiveness units. The two centres, MDT1 and MDT2, are both UK tertiary head and neck oncology departments.

Patient information was anonymized using the National Health Service (NHS) trust identification number and patient NHS numbers. Information was collected on tumour site, date of diagnosis, TNM staging before and after treatment, treatment received, tumour depth on pre-treatment imaging, timing of the biopsy related to pre-treatment imaging, tumour depth on final histopathology, malignant recurrences, and any significant clinical outcomes during the time period.

The primary sample drawn from the MDT databases identified 5120 patients, of whom 4857 were excluded as they were not within the target population. Thirtynine patient cases were removed due to insufficient documentation. Thirteen patients were excluded as histopathology showed tumours of the salivary glands, and 26 patients were excluded as they did not have surgical treatment and therefore did not have a postoperative histopathological specimen for analysis (Fig. 1).

The final sample consisted of 185 patients. Ten patients had undergone previous treatment for oral cavity tumours and one for a tonsillar tumour. The patients were followed up for a mean 26 months (range 1 month to 5 years).

Regarding the imaging and assessment protocols at the two centres, both used combinations of magnetic resonance imaging (MRI), ultrasound (US), computed tomography (CT), incisional biopsy, and ultrasound-guided fine needle aspiration (USFNA). At MDT1, the oral cavity assessment was performed by MRI head and

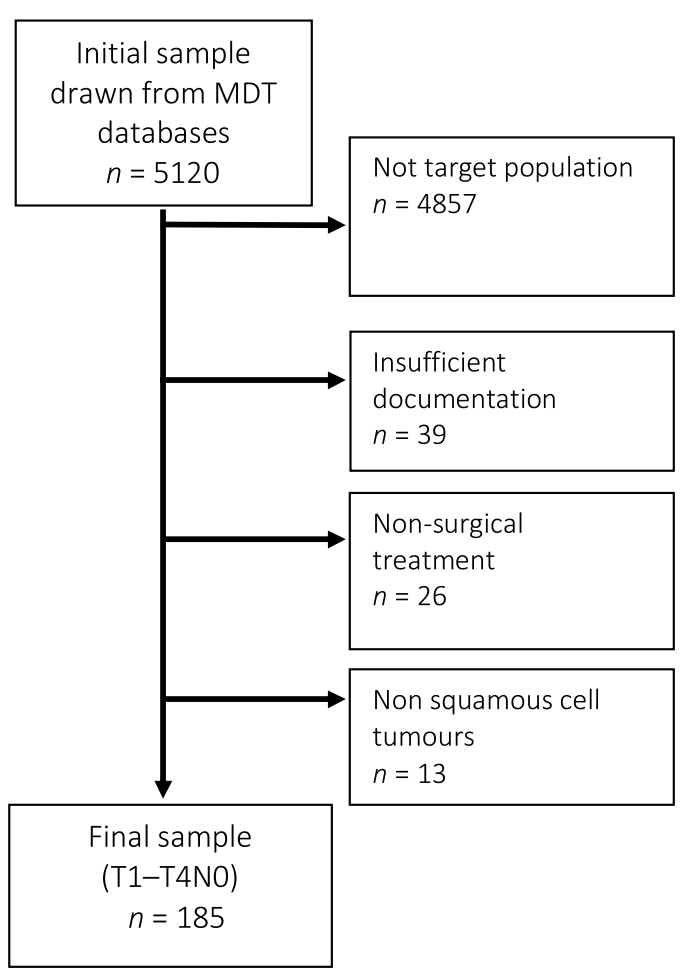

Fig. 1. Flow diagram of study inclusions and exclusions. neck + intraoral US + incisional biopsy; neck node assessment was performed by MRI head and neck \pm USFNA if there was a palpable lump; chest staging was done by CT chest. At MDT2, the oral cavity assessment was performed by MRI or CT head and neck + incisional biopsy; neck node assessment was performed by MRI or CT head and neck \pm USFNA if there was a palpable lump; chest staging was done by $\mathrm{CT}$ chest. The imaging protocol at the two MDT centres differed slightly due to resource availability. This provided the opportunity to compare assessments of preoperative tumour depth made from three different imaging modalities.

The minimum requirement for oral cavity assessment at these centres is either MRI or CT, as per the British Association of Head and Neck Oncologists (BAHNO) ${ }^{2}$ and National Institute for Health and Care Excellence (NICE) guidelines ${ }^{10}$. In the study sample, 12 patients did not receive MRI and instead received CT with contrast. Reasons included claustrophobia, implanted metalwork, and inability to recline flat for the duration of the scan. Nine patients received neither MRI nor CT, only intraoral US (IOUS), mainly due to contrast allergy.

Routine MRI sequences included T1, STIR, T2, and axial T1-weighted sequences pre- and post-contrast (the 
post-contrast sequence included fat suppression). Measurements were preferentially done on coronal gadoliniumenhanced T1-weighted images (3-mm slice thickness). Multiple MRI scanners were used at the two centres, primarily 3.0 T Philips (Philips Medical Systems, Bothell, WA, USA). CT was performed with Siemens scanners using contrast (Siemens Healthcare, Erlangen, Germany). A delay of 90 seconds with multiplanar reformats with soft tissue window setting and dedicated bone algorithm was used, with maximum 1-mm slice thickness.

The radiological analysis of pre-treatment tumour depth was drawn from pretreatment radiology reports. These were finalized and validated at weekly head and neck MDT meetings. In cases where tumour depth was not specified in the reports, one named head and neck radiologist at each centre provided a blinded analysis. Radiological tumour depth assessment on CT and MRI was performed in the same fashion as described by Lam et al. ${ }^{11}$. A horizontal line joining two tumour mucosae junctions was drawn as a reference line, and depth was measured from this line to the point of maximal tumour invasion. Tumour depth was measured perpendicular to the lateral aspect of the oral tumour margin on both the contrast-enhanced CT or MRI and the T2/ STIR sequences.

Measurements from IOUS were performed similarly, with a line drawn perpendicular to the mucosal surface, measuring the deepest point of abnormality. No local anaesthetic was used for IOUS and minimal pressure was applied. IOUS was performed with a General Electric L8-
18 hockey stick probe with a $5-9-\mathrm{MHz}$ frequency range and a $25-\mathrm{mm}$ footprint and ultrasound gel.

Histopathological analysis of tumour depth of invasion was taken from the final histopathology reports made by six named head and neck pathologists. This was considered to be the 'gold standard' assessment of depth of invasion for the purpose of analysis.

Data were collected into a passwordprotected Microsoft Excel spreadsheet (2019). The multivariate analysis was undertaken by a medical statistician using $\mathrm{R}^{12}$.

Treatment and outcome data were also analysed for this study, in order to contextualize the accuracy of the imaging modalities in the assessment of tumour depth of invasion. These measurements are used to make important clinical decisions at MDT meetings, so this highlights the impact of making accurate measurements from preoperative imaging.

The aim of this study was to establish the accuracy of assessments of tumour depth of invasion by comparing the pretreatment depths on imaging with the final histopathological tumour depths.

\section{Results}

The histopathological tumour depths in this sample did not show a normal distribution (Fig. 2). Most tumours (76.2\%) had depths of $<9 \mathrm{~mm}$.

Overall, 162 MRI scans, 40 CT scans, and 84 ultrasound scans were performed and analysed for this sample.

In $35.8 \%$ of cases scanned with MRI, no measurable lesion could be identified (mean depth $3.7 \mathrm{~mm}$, range $0-23 \mathrm{~mm}$ ), of which $75.9 \%$ were $\mathrm{T} 1$ tumours. In $47.5 \%$ of tumours scanned with $\mathrm{CT}$, a measurable lesion could not be identified (mean depth $6.6 \mathrm{~mm}$, range $0-19 \mathrm{~mm}$ ) of which $57.9 \%$ were T1 tumours. Of those cases scanned with IOUS, $7.1 \%$ failed to identify the lesion, of which $50 \%$ were $\mathrm{T} 1$ tumours (mean depth $5.2 \mathrm{~mm}$, range 0$16 \mathrm{~mm}$ ) (Table 1). Four MRI scans and four CT scans failed to identify lesions that were $\geq 10 \mathrm{~mm}$ in depth, which may indicate that these images were subject to substantial artefacts from dental restorations.

A minority of scans were performed prior to diagnostic biopsy $(21.6 \%$ of MRI, $27.5 \%$ of CT, and $23.8 \%$ of IOUS), and a comparison of the accuracy was made between scans performed before and after biopsy (Table 1). An arbitrary margin of $3 \mathrm{~mm}$ from the histopathological measurement was applied as an acceptable level of variance from the 'gold standard'. No statistically significant differences were found in this study between scans done before and after biopsy, for any of the three imaging modalities (MRI, $P=0.36$; CT, $P=0.35$; US, $P=0.11$ ).

Of the measurements made from scans performed prior to biopsy (with visible lesions), $60.9 \%$ overestimated the tumour depth of invasion, and of those scans done after biopsy, $53.7 \%$ overestimated tumour depth of invasion.

\section{MRI}

The depth assessment was found to be accurate to within $\pm 3 \mathrm{~mm}$ of the histopathological depth in $55.8 \%$ of tumours

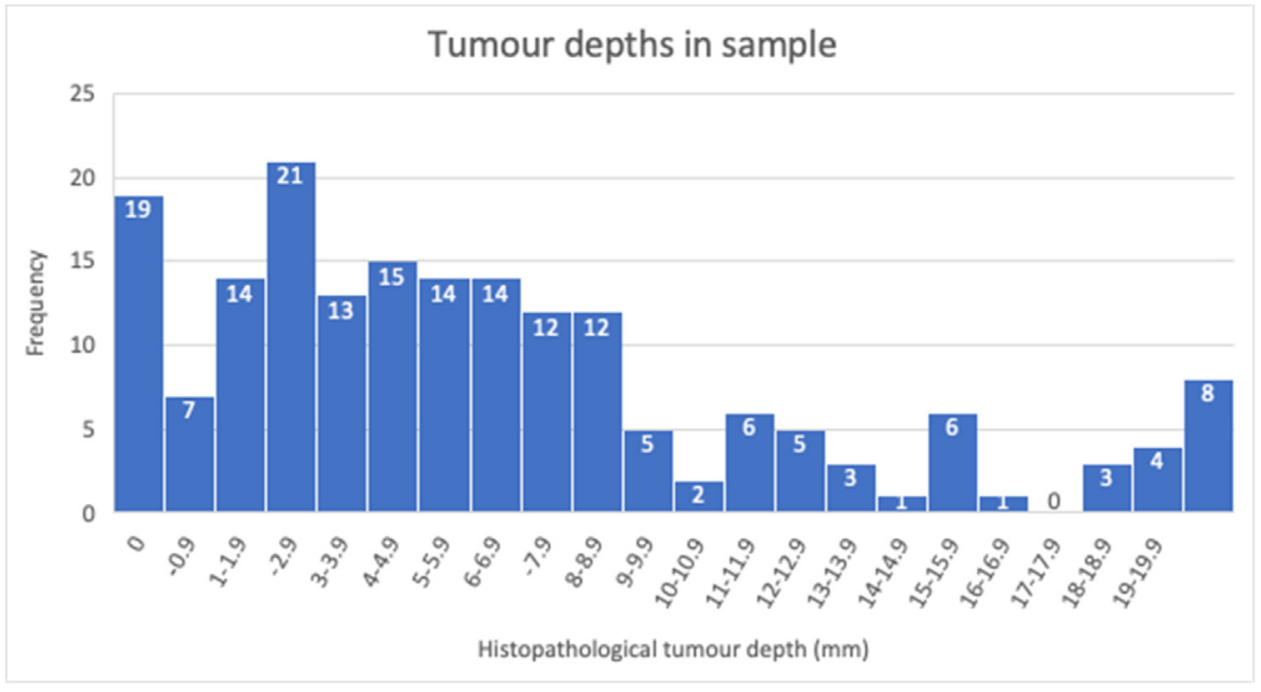

Fig. 2. Histopathological tumour depths for the study sample $(N=185)$. 
Table 1. Overall accuracy of each imaging modality, and relation to timing of biopsy.

\begin{tabular}{|c|c|c|c|c|}
\hline $\begin{array}{l}\text { Type of } \\
\text { scan }\end{array}$ & $\begin{array}{l}\text { Number in } \\
\text { sample }\end{array}$ & $\begin{array}{l}\text { Number (\%) of scans } \\
\text { in sample with no } \\
\text { visible lesion }\end{array}$ & $\begin{array}{l}\text { Number (\%) done } \\
\text { before biopsy }\end{array}$ & $\begin{array}{l}\text { Number (\%) pre-biopsy scan } \\
\text { (with visible lesion) with accurate depth } \\
\text { assessment to within } 3 \mathrm{~mm} \\
\text { of histopathological depth }\end{array}$ \\
\hline MRI & 162 & $58(35.8)$ & 35 (21.6) & $\begin{array}{l}11(31.4) \\
(P=0.36)\end{array}$ \\
\hline CT & 40 & $19(47.5)$ & $11(27.5)$ & $\begin{array}{l}3(27.3) \\
(P=0.35)\end{array}$ \\
\hline US (intraoral) & 84 & $6(7.1)$ & $20(23.8)$ & $\begin{array}{l}15(75) \\
(P=0.11)\end{array}$ \\
\hline
\end{tabular}

MRI, magnetic resonance imaging; CT, computed tomography; US, ultrasound.

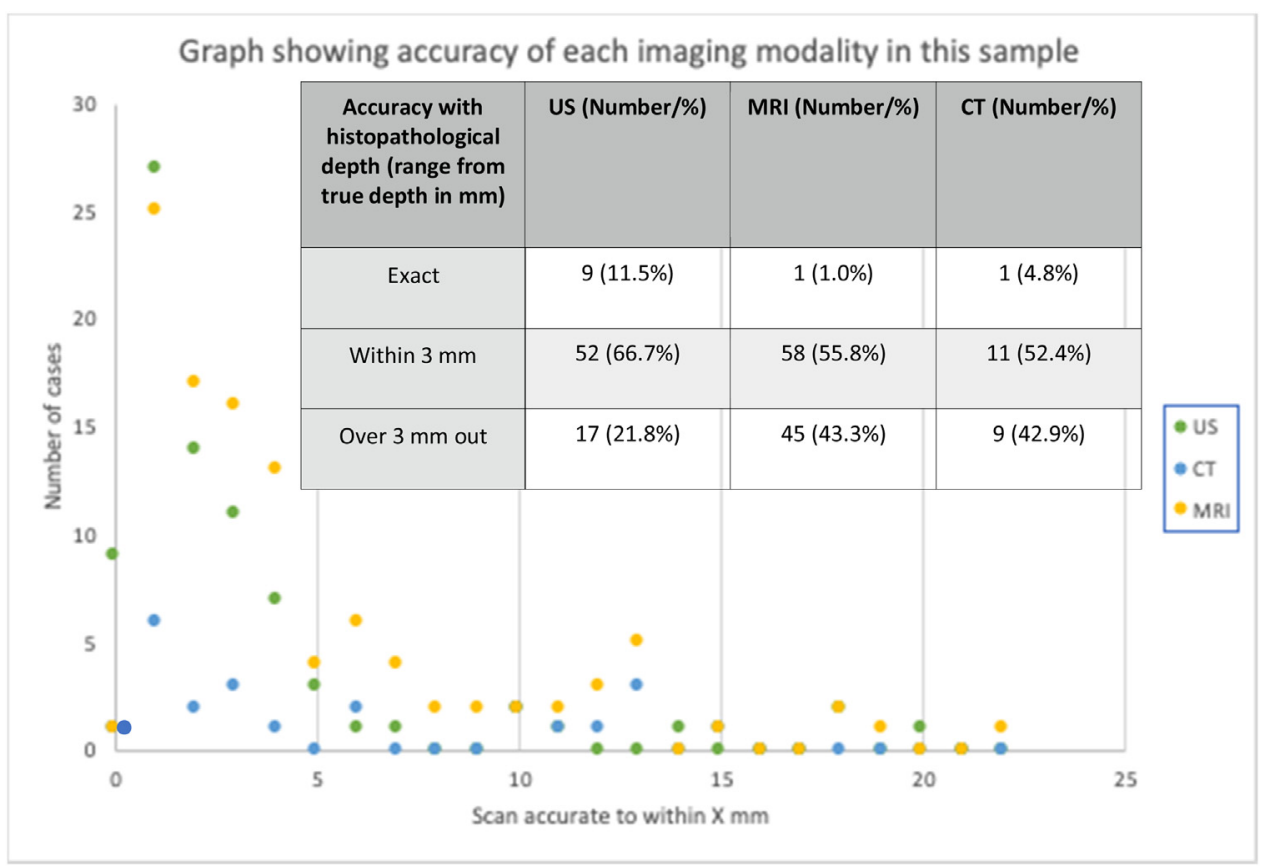

Fig. 3. Scatter plot showing the accuracy of each imaging modality.

scanned with MRI, while one MRI scan $(1.0 \%)$ in this sample made an exact assessment of the tumour thickness; $43.3 \%$ of MRI scans were more than $3 \mathrm{~mm}$ variant from the histopathological depth (Fig. 3). The total percentage of measurements that were accurate or within $3 \mathrm{~mm}$ was $56.7 \%$. Of the MRI scans performed prior to biopsy, $31.4 \%$ were accurate to within $3 \mathrm{~mm}$ of the histopathological depth $(P=0.36)$ (Table 1).

\section{CT}

One CT scan (4.8\%) was exactly accurate with the histopathological tumour depth. Of the remaining CT scans, $52.4 \%$ were within $3 \mathrm{~mm}$ of the histopathological depth and $42.9 \%$ were more than $3 \mathrm{~mm}$ variant from the histopathological depth
(Fig. 3). The total percentage of measurements that were accurate or within $3 \mathrm{~mm}$ was $57.1 \%$. Of the CT scans performed prior to biopsy, $27.3 \%$ were accurate to within $3 \mathrm{~mm}$ of the histopathological depth $(P=0.35)$ (Table 1).

\section{IOUS}

Tumours scanned with IOUS were exactly accurate with the true tumour depth in $11.5 \%$ of cases, and a further $66.7 \%$ were within $3 \mathrm{~mm}$ of the true depth; $21.8 \%$ of cases were more than $3 \mathrm{~mm}$ variant from the histopathological depth (Fig. 3). The total percentage of measurements that were accurate or within $3 \mathrm{~mm}$ was $78.2 \%$. Of the IOUS scans performed prior to biopsy, $75 \%$ were accurate to within
$3 \mathrm{~mm}$ of the histopathological depth $(P=0.11)$ (Table 1).

\section{Comparison of imaging modalities}

Differences in accuracy between the imaging modalities were assessed for statistical significance. This was done by calculating the average difference between histopathological tumour depth and radiological tumour depth for each modality. The Student $t$-test was used for this analysis. Scans in which lesions could not be identified were excluded from the evaluations of accuracy, as no comparison could be made with the histopathology results.

No significant difference was found between CT and MRI $(P=0.06)$, or between CT and IOUS $(P=0.47)$. The difference 
Table 2. Tumour location and accuracy by modality.

\begin{tabular}{|c|c|c|c|c|c|c|}
\hline Tumour site & $\begin{array}{l}\text { Number US done } \\
\text { (with visible lesion) }\end{array}$ & $\begin{array}{l}\text { Number (\%) US } \\
\text { (with visible lesion) } \\
\text { accurate to } \\
\text { within } 3 \mathrm{~mm}^{\mathrm{a}}\end{array}$ & $\begin{array}{l}\text { Number MRI done } \\
\text { (with visible lesion) }\end{array}$ & $\begin{array}{l}\text { Number (\%) MRI } \\
\text { (with visible lesion) } \\
\text { accurate to } \\
\text { within } 3 \mathrm{~mm}^{\mathrm{a}}\end{array}$ & $\begin{array}{l}\text { Number CT } \\
\text { done (with } \\
\text { visible lesion) }\end{array}$ & $\begin{array}{l}\text { Number (\%) CT } \\
\text { (with visible lesion) } \\
\text { accurate to } \\
\text { within } 3 \mathrm{~mm}^{\mathrm{a}}\end{array}$ \\
\hline FOM & 10 & $8(80 \%)$ & 7 & $4(57.1 \%)$ & 1 & $0(0 \%)$ \\
\hline Hard palate & 5 & $4(80 \%)$ & 5 & $4(60 \%)$ & 1 & $1(100 \%)$ \\
\hline Buccal mucosa & 11 & $9(81.8 \%)$ & 20 & $13(65 \%)$ & 3 & $2(66.7 \%)$ \\
\hline Tongue & 47 & $38(80.9 \%)$ & 57 & $30(52.6 \%)$ & 8 & $4(50 \%)$ \\
\hline Gingival mucosa & 5 & $2(40 \%)$ & 15 & $8(53.3 \%)$ & 8 & $5(62.5 \%)$ \\
\hline
\end{tabular}

US, ultrasound; MRI, magnetic resonance imaging; CT, computed tomography; FOM, floor of the mouth.

${ }^{a}$ Includes exact cases and cases within $3 \mathrm{~mm}$.

between MRI and IOUS was found to be statistically significant $(P<0.001)$.

Variation in accuracy was seen by anatomical site of the tumour (see Table 2). The most accurate site was the hard palate (mean histopathological depth $3.9 \mathrm{~mm}$, range 0 $16 \mathrm{~mm}$ ), with accuracy to within $3 \mathrm{~mm}$ of the histopathological depth for $100 \%$ of tumours scanned with $\mathrm{CT}, 80 \%$ scanned with IOUS, and $60 \%$ with MRI. The least accurate site was floor of the mouth (mean histopathological depth $3.9 \mathrm{~mm}$, range 0 $14 \mathrm{~mm}$ ), with $80 \%$ of IOUS measurements, $57.1 \%$ of MRI measurements, and $0 \%$ of CT measurements found to be accurate to within $3 \mathrm{~mm}$ of the histopathological depth. Of course, it must be acknowledged that some of the differences in accuracy of the different imaging modalities reflect the inherent strengths and limitations of the individual techniques; for example CT performs well for bony structure analysis, as seen in the hard palate.

The statistical analysis of the results for the anatomical sites did not reach statistical significance (Fisher's exact test, $P=0.34)$.

\section{Accuracy in $\mathrm{T}$ staging cases}

Data were analysed to determine how often each type of scan accurately determined the tumour $\mathrm{T}$ stage. Cases were assessed for changes between pre-treatment and the definitive $\mathrm{T}$ grade, and the imaging techniques used in each case were analysed (Fig. 4).

In order to reflect clinical practice, data were further analysed to assess the effect of the double or triple radiological staging assessment that each case received. A mosaic plot was created to depict this (Fig. 5). This shows that $82 \%$ of $\mathrm{T} 1$ tumours and $65 \%$ of $\mathrm{T} 4$ tumours were correctly staged, and $50 \%$ of T2 tumours were correctly staged (Fig. 5).

\section{Surgical management of the neck}

Eighty-five patients (45.9\%) underwent clinical observation of the neck (mean tumour depth $4.3 \mathrm{~mm}$, range $0-19 \mathrm{~mm}$ ). The clinical observation protocol was as per BAHNO guidelines ${ }^{2}$, with examination every $1-3$ months in years 1 and 2 , every 3-6 months in years 3-5, and tailored radiological investigations in response to clinical suspicion. Neck node recurrences were seen in $10(11.8 \%)$ of these patients (Table 3).

Seventy-five patients $(40.5 \%)$ received an END (mean tumour depth $9.9 \mathrm{~mm}$, range 0-28 mm) of whom four (5.3\%) developed neck node recurrences (Table 3 ).

Nineteen patients $(10.3 \%)$ underwent sentinel node biopsy (SNB), of whom four $(21.1 \%)$ were positive (mean tumour depth $3.7 \mathrm{~mm}$, range $0-15 \mathrm{~mm})$. One of these patients $(5.3 \%)$ developed neck node recurrences (Table 3 ).

A further 80 patients $(43.2 \%)$ would have been eligible for SNB based on 2016 NICE guidance (cT1-T2N0, seventh edition of the TNM classification) ${ }^{10}$. Of these, $46(57.5 \%)$ underwent clinical observation and four $(8.7 \%)$ developed neck node metastases. In this group, the mean radiological depth was $8.9 \mathrm{~mm}$ (range 0 $27 \mathrm{~mm}$ ) and the mean pathological depth was $4.0 \mathrm{~mm}$ (range $0-15 \mathrm{~mm}$ ). The remaining 34 patients received an END (mean tumour pathological depth $7.4 \mathrm{~mm}$,

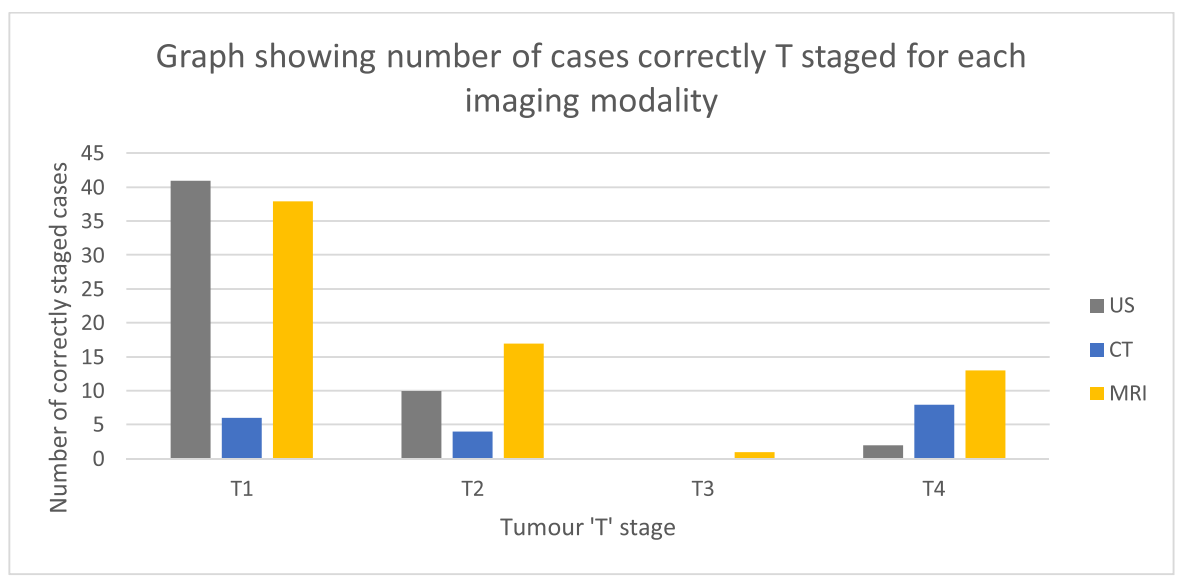

Fig. 4. Grouped bar chart showing correct $\mathrm{T}$ staging in relation to each type of imaging. 


\section{Mosaic plot comparıng preoperative staging to postoperative staging (TNM8) on depth alone}

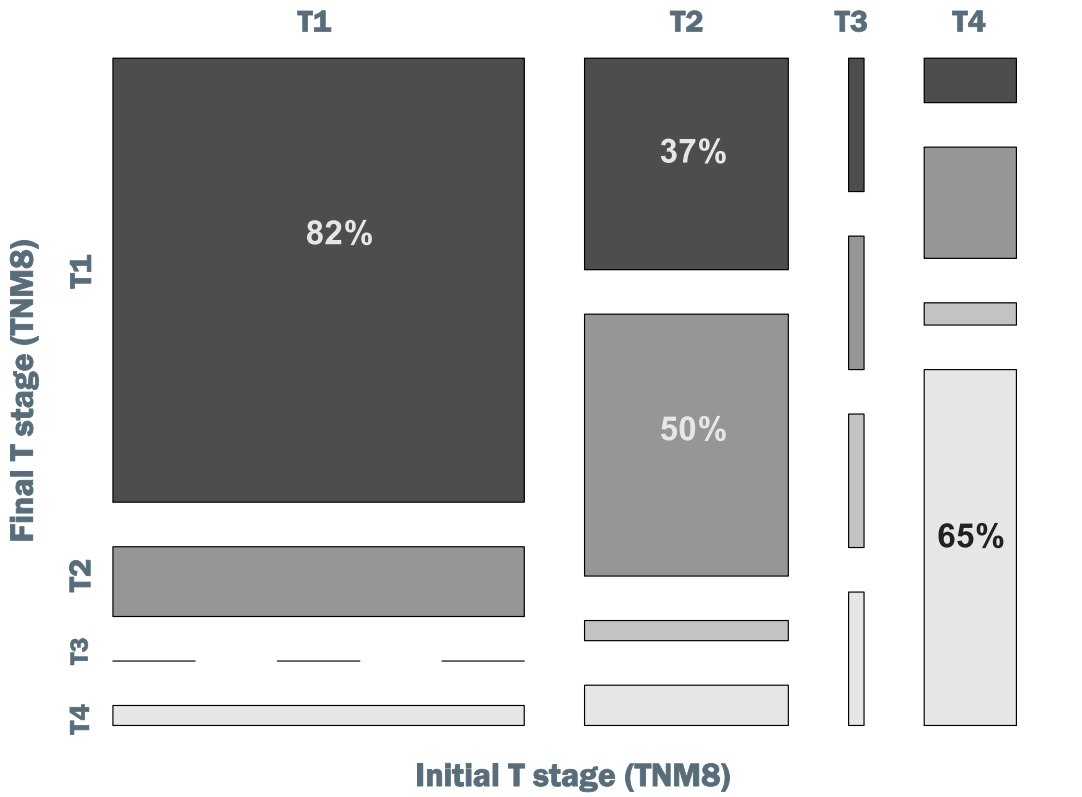

Fig. 5. Mosaic plot comparing preoperative and postoperative $\mathrm{T}$ stages, combining all imaging modality types.

Table 3. Neck management, positive neck rate, and neck relapse rate.

\begin{tabular}{|c|c|c|c|}
\hline Neck management & $\begin{array}{l}\text { Mean pathological } \\
\text { tumour depth (mm) }\end{array}$ & Node-positive neck rate $(\%)$ & Neck relapse rate $(\%)$ \\
\hline $\begin{array}{l}\text { Elective neck dissection } \\
(n=75)\end{array}$ & 9.91 & $\begin{array}{l}28 \\
(n=21)\end{array}$ & $\begin{array}{l}5.3 \\
(n=4)\end{array}$ \\
\hline $\begin{array}{l}\text { Clinical observation } \\
(n=85)\end{array}$ & 4.34 & - & $\begin{array}{l}11.8 \\
(n=10)\end{array}$ \\
\hline $\begin{array}{l}\text { Sentinel node biopsy } \\
(n=19)\end{array}$ & 3.71 & $\begin{array}{l}21.1 \\
(n=4)\end{array}$ & $\begin{array}{l}5.3 \\
(n=1)\end{array}$ \\
\hline
\end{tabular}

range $0-25.5 \mathrm{~mm})$; two of these patients $(5.9 \%)$ developed neck node recurrences.

The difference between the mean pathological tumour depths of these two clinical observation and END groups reached statistical significance $(P=0.002)$.

Six patients $(3.2 \%)$ received wide local excision of the tumour followed by primary radiotherapy (mean tumour depth $7.0 \mathrm{~mm}$, range $1.5-12 \mathrm{~mm}$ ).

\section{Discussion}

The most striking finding of this study is that despite following imaging guidelines, the preoperative assessment of tumour depth differed by over $3 \mathrm{~mm}$ from the 'gold standard' histopathological measurement in $21.8 \%$ of IOUS, $43.3 \%$ of MRI, and $42.9 \%$ of CT (Fig. 3). Resection specimens can shrink due to factors like dehydration, and the magnitude varies with stage and site $^{13}$, but clearly there are implications of using tumour depth of invasion from preoperative scans to decide treatment. Data on surgical management of the neck (above) and changes to definitive staging (Fig. 5) shown in this study may point towards patients being over-treated and incurring additional morbidity.

This retrospective audit gives a review of the actual head and neck imaging protocol at two tertiary UK head and neck cancer centres from 2014 to 2018 . Due to the retrospective design, this study was not randomized, and the neck management and outcome data need careful interpretation.

Surgical neck management in these cN0 cases would have been based on an arbitrary depth cut-off and would typically have been multifactorial, taking into account surgical concerns such as neck access for reconstruction, as well as patient choice and performance status.

SNB at these centres was not used routinely until after the 2016 NICE guidelines were published ${ }^{10}$; at first TNM7 was adhered to, and currently TNM8 is followed (for cT1-T2N0 cases only). Much of the data were gathered prior to the work of D'Cruz ${ }^{6}$, publication of the NICE 2016 guidelines $^{10}$, and the UK SEND study ${ }^{14}$, all of which recommend END to confer a survival advantage. Had the study period started after publication of TNM $8^{1}$, it is possible that some cases may have been managed differently. Certainly, for T3T4N0 cases, we strongly advocate END.

The results showed that IOUS currently provides the most accurate preoperative assessment of tumour depth, particularly 
in thin tumours ( $<5 \mathrm{~mm}$ depth) and tumours of the mobile tongue. This is in keeping with the literature. A comparable retrospective study by Shintani et al. ${ }^{15}$ found measurements of tumour depth made preoperatively with IOUS to be significantly correlated with histological measurements (Pearson's $r=0.988$ ), and to be superior to CT and MRI measurements, but for sites limited to the tongue, buccal mucosa, and floor of the mouth. Yuen et al. ${ }^{16}$, in a study of 45 tongue carcinomas, found that IOUS depth measurements taken by the surgeon immediately prior to glossectomy were significantly correlated with histopathological depth $(P<0.005)$.

The recent literature has stated that standard MRI does not adequately predict histopathological tumour depth, calling for a re-evaluation of MRI in tumour assessment ${ }^{17}$. The present study data showed MRI to be most accurate for the deepest tumours, again consistent with the current literature, which has found preoperative MRI assessment to be most accurate with tumours $>5 \mathrm{~mm}$ in depth ${ }^{18,19}$.

It must be stated that the ability of imaging to identify a measurable lesion is vulnerable to artefacts. Metal artefacts, such as dental amalgam, can obscure structures and decrease the diagnostic value. Diagnostic value is also subject to timing related to surgical intervention, as biopsy or excision will cause inflammation and can lead to discrepancies in measurements. Biopsy prior to staging scans is, however, common in the clinical setting in order to avoid unnecessary irradiating imaging until the malignancy is confirmed, although to preserve measurement accuracy it is preferable to biopsy after imaging. The study data did not show that timing of biopsy related to imaging altered the measurement accuracy $(P=$ 0.39 ). It was also observed that $70 \%$ of scans done prior to biopsy were for tumours $\leq 10 \mathrm{~mm}$ depth, perhaps indicating that scans may be expedited for thinner lesions with an uncertain diagnosis.

Despite varying numbers according to the anatomical site, all sites were included in the results. Given that there is no difference in staging based on site for oral cavity tumours, we feel it remains important to be able to assess depth of invasion to make clinical decisions, and it may be that different modalities work better for different sites.

In the future, techniques such as optical coherence tomography (OCT) could be used to provide an 'optical biopsy' and thereby prevent the potential for radiographic artefacts caused by biopsy.
However, the recent literature on OCT for oral tissues indicates that this is not yet reliable ${ }^{21}$.

In conclusion, current imaging protocols may not provide robust and accurate assessment of tumour depth in the oral cavity. The most reliable method found in this study was IOUS, and we recommend greater uptake of this still novel technique for staging of head and neck tumours.

\section{Funding}

None.

\section{Competing interests}

None.

\section{Ethical approval}

Ethics permission was not required.

\section{Patient consent}

Patient consent was not required.

\section{References}

1. Brierley JD, Gospodarowicz MK, Wittekind C. The TNM classification of malignant tumours. eighth edition. Union for International Cancer Control; 2016.

2. British Association of Head and Neck Oncologists. BAHNO standards. London: BAHNO; 2009.

3. International Consortium for Outcome Research (ICOR) in Head and Neck Cancer. Ebrahimi A, Gil Z, Amit M, Yen TC, Liao CT, Chaturvedi P, Agarwal JP, Kowalski LP, Kreppel M, Cernea CR, Brandao J, Bachar G, Bolzoni Villaret A, Fliss D, Fridman E, Robbins KT, Shah JP, Patel SG, Clark JR. Primary tumour staging for oral cancer and a proposed modification incorporating depth of invasion: an international multicenter retrospective study. JAMA Otolaryngol Head Neck Surg 2014;140:1138-48.

4. Yuen AP, Lam KY, Wei WI, Lam KY, Ho CM, Chow TL, Yuen WF. A comparison of the prognostic significance of tumor diameter, length, width, thickness, area, volume, and clinicopathological features of oral tongue carcinoma. Head Neck 2002;24:513-20.

5. de Matos LL, Manfro G, Santos RV, Stabenow E, Mello ES, Alves VA, Pinto FR, Kulcsar MA, Brandão LG, Cernea CR. Tumor thickness as a predictive factor of lymph node metastasis and disease recurrence in $\mathrm{T} 1 \mathrm{~N} 0$ and T2N0 squamous cell carcinoma of the oral tongue. Oral Surg Oral Med Oral Pathol 2014;118:209-17.

6. D'Cruz AK, Vaish R, Kapre N, Dandekar M, Gupta S, Hawaldar R, Agarwal JP, Pantvaidya G, Chaukar D, Deshmukh A, Kane S,
Arya S, Ghosh-Laskar S, Chaturvedi P, Pai P, Nair S, Nair D, Badwe R. Elective versus therapeutic neck dissection in node-negative oral cancer. N Engl J Med 2015;373:521-9.

7. National Cancer Institute. Lip and oral cavity cancer treatment (adult) (PDQ). PDQ Cancer Information Summaries [Internet]. NCBI Bookshelf, 2018. https://www.ncbi.nlm.nih. gov/books/NBK65821/ [Accessibility verified October 19, 2020].

8. Huang SH, Hwang D, Lockwood G, Goldstein DP, O'Sullivan B. Predictive value of tumor thickness for cervical lymph-node involvement in squamous cell carcinoma of the oral cavity. Cancer 2009;115:1489-97.

9. National Comprehensive Cancer Network. NCCN clinical practice guidelines in oncology (NCCN guidelines)—head and neck cancers. Pennsylvania, USA: NCCN; 2019.

10. National Institute for Health and Care Excellence. Cancer of the upper aerodigestive tract: assessment and management in people aged 16 and over (NG36). London, UK: National Collaborating Centre for Cancer; 2016.

11. Lam P, Au-Yeung KM, Cheng PW, Wei WI, Yuen AP, Trendell-Smith N, Li JH, Li R. Correlating MRI and histologic tumor thickness in the assessment of oral tongue cancer. AJR Am J Roentgenol 2004;182:803-8.

12. $\mathrm{R}$ Core Team. $R$ : A language and environment for statistical computing. Vienna, Austria: R Foundation for Statistical Computing; 2019.

13. Shah A. Postoperative pathologic assessment of surgical margins in oral cancer: a contemporary review. J Oral Maxillofac Pathol 2018;22:78-85.

14. Hutchison IL, Ridout F, Cheung SMY, Shah N, Hardee P, Surwald C, Thiruchelvam J, Cheng L, Mellor TK, Brennan PA, Baldwin AJ, Shaw RJ, Halfpenny W, Danford M, Whitley S, Smith G, Bailey MW, Woodwards B, Patel M, McManners J, Chan $\mathrm{CH}$, Burns A, Praveen P, Camilleri AC, Avery C, Putnam G, Jones K, Webster K, Smith WP, Edge C, McVicar I, Grew N, Hislop S, Kalavrezos N, Martin IC, Hackshaw A. Nationwide randomised trial evaluating elective neck dissection for early stage oral cancer (SEND study) with meta-analysis and concurrent real-world cohort. $\mathrm{Br} \mathrm{J}$ Cancer 2019;121:827-36.

15. Shintani S, Yoshihama Y, Ueyama Y, Terakado N, Kamei S, Fijimoto Y, Hasegawa Y, Matsuura H, Matsumura T. The usefulness of intraoral ultrasonography in the evaluation of oral cancer. Int J Oral Maxillofac Surg 2001;30:139-43.

16. Yuen APW, Ng RWM, Lam PKY, Ho A. Preoperative measurement of tumor thickness of oral tongue carcinoma with intraoral ultrasonography. Head Neck 2008;30:230-4.

17. Lwin CT, Hanlon R, Lowe D, Brown JS, Woolgar JA, Triantafyllou A, Rogers SN, Bekiroglu F, Lewis-Jones H, Wieshmann $\mathrm{H}$, Shaw RJ. Accuracy of MRI in prediction 
8 Cocker et al.

of tumour thickness and nodal stage in oral squamous cell carcinoma. Oral Oncol 2012;48:149-54.

18. Alsaffar HA, Goldstein DP, King EV, de Almeida JR, Brown DH, Gilbert RW, Gullane PJ, Espin-Garcia O, Xu W, Irish JC. Correlation between clinical and MRI assessment of depth of invasion in oral tongue squamous cell carcinoma. J Otolaryngol Head Neck Surg 2016;45:61. http://dx.doi. org/10.1186/s40463-016-0172-0.

19. Goel V, Parihar PS, Parihar A, Goel AK, Waghwani K, Gupta R, Bhutekar U. Accu- racy of MRI in prediction of tumour thickness and nodal stage in oral tongue and gingivobuccal cancer with clinical correlation and staging. $J$ Clin Diagn Res 2016;10. http://dx.doi.org/10.7860/JCDR/2016/ 17411.7905. TC01-TC05.

20. Fujimoto JG, Pitris C, Boppart SA, Brezinski ME. Optical coherence tomography: an emerging technology for biomedical imaging and optical biopsy. Neoplasia 2000;2:9-25. http://dx.doi.org/10.1038/sj.neo.7900071.

21. Gentile E, Maio C, Romano A, Laino L, Lucchese A. The potential role of in vivo optical coherence tomography for evaluating oral soft tissue: a systematic review. $J$ Oral Pathol Med 2017;46:864-76.

Address:

Hana Cocker

University College London Hospital

235 Euston Rd

Bloomsbury

London NW1 2BU

UK

E-mail: hana.cocker@gmail.com 